\title{
Redox signaling regulated by electrophiles and reactive sulfur species
}

\author{
Motohiro Nishida, ${ }^{1,2,3}$ Yoshito Kumagai, ${ }^{4}$ Hideshi Ihara, ${ }^{5}$ Shigemoto Fujii, ${ }^{6}$ Hozumi Motohashi ${ }^{7}$ and Takaaki Akaike,* \\ 'Division of Cardiocirculatory Signaling, Okazaki Institute for Integrative Bioscience (National Institute for Physiological Sciences), \\ National Institutes of Natural Sciences, 5-1 Higashiyama, Myodaiji-cho, Okazaki 444-8787, Japan \\ 2Department of Translational Pharmaceutical Sciences, Graduate School of Pharmaceutical Sciences, Kyushu University, \\ 3-1-1 Maidashi, Higashi-ku, Fukuoka 812-8582, Japan \\ ${ }^{3}$ PRESTO, Japan Science and Technology Agency (JST), 4-1-8 Honcho, Kawaguchi 332-0012, Japan \\ ${ }^{4}$ Environmental Biology Section, Faculty of Medicine, University of Tsukuba, 1-1-1 Tennodai, Tsukuba 305-8575, Japan \\ ${ }^{5}$ Department of Biological Science, Graduate School of Science, Osaka Prefecture University, 1-1 Gakuen-cho, Naka-ku, Sakai 599-8531, Japan \\ ${ }^{6}$ Department of Environmental Health Sciences and Molecular Toxicology, Tohoku University Graduate School of Medicine, \\ 2-1 Seiryo-machi, Aoba-ku, Sendai 980-8575, Japan \\ 'Department of Gene Expression Regulation, Institute of Development, Aging and Cancer, Tohoku University, \\ 4-1 Seiryo-machi, Aoba-ku, Sendai 980-8575, Japan
}

(Received 5 September, 2015; Accepted 10 September, 2015; Published online 17 February, 2016)

Redox signaling is a key modulator of oxidative stress induced by nonspecific insults of biological molecules generated by reactive oxygen species. Current redox biology is revisiting the traditional concept of oxidative stress, such that toxic effects of reactive oxygen species are protected by diverse antioxidant systems upregulated by oxidative stress responses that are physiologically mediated by redox-dependent cell signaling pathways. Redox signaling is thus precisely regulated by endogenous electrophilic substances that are generated from reactive oxygen species and nitric oxide and its derivative reactive species during stress responses. Among electrophiles formed endogenously, 8-nitroguanosine 3 ',5'-cyclic monophosphate (8-nitro-cGMP) has unique cell signaling functions, and pathways for its biosynthesis, signaling mechanism, and metabolism in cells have been clarified. Reactive sulfur species such as cysteine hydropersulfides that are abundant in cells are likely involved in 8-nitro-cGMP metabolism. These new aspects of redox biology may stimulate innovative and multidisciplinary research in cell and stem cell biology; infectious diseases, cancer, metabolic syndrome, ageing, and neurodegenerative diseases; and other oxidative stress-related disorders. This review focuses on the most recent progress in the biosynthesis, cell signaling, and metabolism of 8-nitro-cGMP, which is a likely target for drug development and lead to discovery of novel therapeutics for many diseases.

Key Words: electrophilic signaling, 8-nitro-cGMP, NO, redox signaling, ROS signaling

$\mathrm{T}$ he chemical biology of molecular oxygen and reactive oxygen species (ROS) is evolving as a frontier of research in energy metabolism, cell signaling, gene transcription, host defense, mutagenesis/carcinogenesis, and oxidative stress. Because of its oxygen-dependent redox properties, ROS can regulate physiological functions of various proteins, which may then modulate enzyme reactions involving cellular metabolism and genomic as well as epigenetic events including transcriptional regulation. Such redox-regulated protein effectors are mostly affected by both translational and posttranslational pathways. In addition to several new protein modifications identified so far, still unidentified redox-mediated protein modifications exist, which may lead to a paradigm-shifting concept of ROS and redox biology and even redox pathology known as oxidative stress.

ROS such as superoxide anion $\left(\mathrm{O}_{2}{ }^{--}\right)$and hydrogen peroxide $\left(\mathrm{H}_{2} \mathrm{O}_{2}\right)$ are reduced metabolites that are physiologically generated from molecular oxygen during mitochondrial respiration or antimicrobial defense responses of hosts during infection of cells and tissues. ROS are harmful agents that mediate oxygen toxicity and in fact are involved in the pathogenesis of many diseases associated with oxidative stress. ${ }^{(1-5)}$ These oxidative stress-related diseases include infections; inflammations; cancer; lifestylerelated diseases and metabolic diseases such as arteriosclerosis and diabetes mellitus; and neurodegenerative disorders such as Alzheimer's disease.

Although expression of ROS-producing enzymes such as NADPH oxidase (Nox) and dual oxidase isoforms by various cell types causes general antimicrobial effects, ${ }^{(6,7)}$ a better understanding of ROS biology now suggests that ROS such as $\mathrm{H}_{2} \mathrm{O}_{2}$ may have much more important physiological functions for cells and tissues, especially in the vasculature and in immune, epithelial, neuronal, and endocrine systems. ${ }^{(8,9)}$ Another key molecule affecting ROS functions is nitric oxide (NO), which serves as a master cell signaling factor that functions in different cells and organs. ${ }^{(10)}$ In view of current evidence of ROS signaling, the canonical mechanism of ROS toxicity has changed drastically and is now evolving.

The present review provides a brief overview of the signaling functions of ROS, with a particular focus on endogenous electrophiles derived from ROS and NO, which may indeed lead to a new concept and shift from conventional ROS toxicology. We also discuss unique antioxidant and nucleophilic properties of reactive cysteine persulfides for redox signal regulation. These reactive sulfur species (RSS), such as cysteine persulfides, are now being increasingly investigated in ROS and redox biology, which should result in the most innovative, well-integrated research on the chemical biology of these reactive small molecules.

\section{Redox Signaling Mechanism}

Electrophilic signal formation. ROS, which are simple, low-molecular-weight inorganic compounds, are formed physiologically as reduced metabolites of molecular oxygen. Because of their chemically reactive and thus mostly unstable nature in biological systems, ROS may need to be converted to other molecules to achieve biologically and physiologically relevant

\footnotetext{
*To whom correspondence should be addressed.

E-mail: takaike@med.tohoku.ac.jp

He received "SFRR Japan Scientific Excellence Award" in 2014 in recognition of his outstanding work.
} 
effects. The specificity of ROS signaling functions also depends on a spatiotemporal property of ROS generation that characterizes the molecular reaction environment. Therefore, identifying the secondary ROS mediators that specifically affects, as signal ligands, particular residues of sensor or effector proteins is extremely important (Fig. 1). The exact mechanisms of specific second messenger formation and its spatiotemporal signal regulation remained unknown, however, until we discovered a nitrated cyclic nucleotide, i.e., 8-nitroguanosine 3',5'-cyclic monophosphate (8-nitro-cGMP), as a unique electrophilic signal molecule, formed from NO and ROS. ${ }^{(11)}$

We now understand the structures and functions and even local-

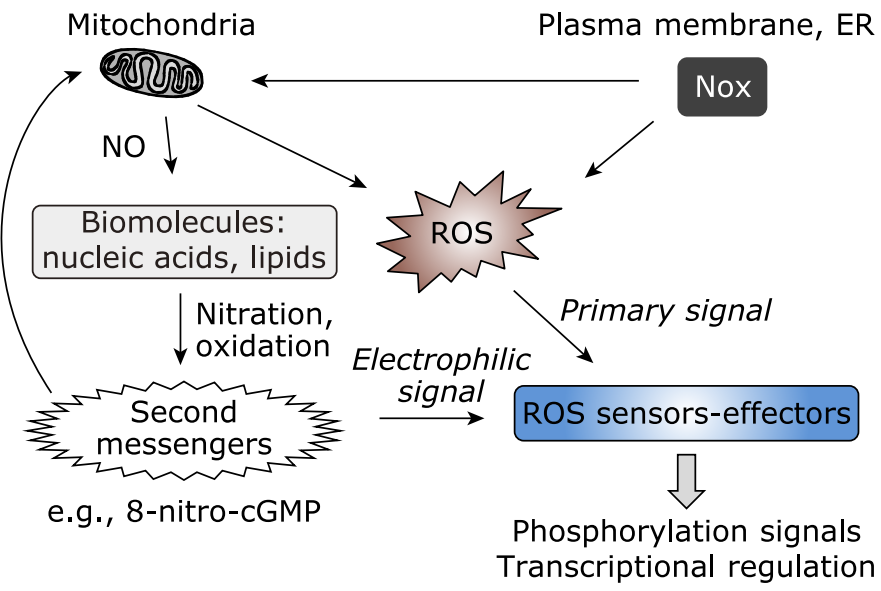

Fig. 1. ROS signaling via formation of electrophilic second messengers ROS produced from different sources, such as Nox in the cytoplasmic membrane or in the endoplasmic reticulum (ER), and mitochondria, are unstable primary signals. ROS themselves, or by means of their reaction with NO, react with various sensor molecules in cells and are converted to more stable electrophilic second messengers such as 8-nitro-cGMP. ization and spatiotemporal dynamics of ROS-producing enzymes (e.g., Nox), the result being that the roles of these enzymes in ROS signaling in cells and tissues are clear. ${ }^{(6,7,12)}$ We specifically clarified an ROS production mechanism that is mediated by cross-talk between Nox and mitochondria, which generates the electrophilic second messenger 8-nitro-cGMP in cells. This 8nitro-cGMP signaling pathway may explain how ROS can transduce their cell signaling in a spatiotemporal manner (Fig. 1). ${ }^{(12)}$ In fact, we identified a specific 8-nitro-cGMP signaling mechanism that affects sensor-effector molecules by means of an electrophilemediated signaling system in cultured cells and in vivo, ${ }^{(13)}$ as described later in this article.

Mechanism of redox sensing. As described above, ROS are unstable with very short half-lives in biological systems. In most ROS signaling processes, such an unstable primary ROS signal is initially transformed into a much more stable secondary messenger such as 8-nitro-cGMP (Fig. 1). During this process, biological molecules, which include nucleic acids, nucleotides, lipids, and reactive protein residues, act as chemical sensors and can effectively perceive ROS, so that they can participate in a wide range of ROS sensing reactions. For example, interaction of ROS and $\mathrm{NO}$, in the presence of nucleotides and fatty acids, results in stable secondary signaling molecules such as 8-nitro-cGMP and nitro-fatty acids. ${ }^{(11,14)}$ In addition, proteins expressing nucleophilic cysteine sulfhydryls as specific redox sensors readily react with ROS or electrophilic second messengers and thereby mediate receptor functions for ROS signaling. Identification of these sensor molecules is therefore essential for understanding the sensing specificity and selectivity of ROS ligands. We and others also extensively studied the structures and functions of sensor and effector proteins modified by an ROS signal or its secondary signaling molecules, with a focus on, for example, 8-nitro-cGMP (Fig. 2). ${ }^{(11-21)}$ Recent discoveries of such unique signal ligands and sensors will clarify the whole picture of diverse ROS sensing and signal transduction mechanisms.

Effector function. Different effectors, as affected by ROS or secondary signaling molecules, are induced during the changes

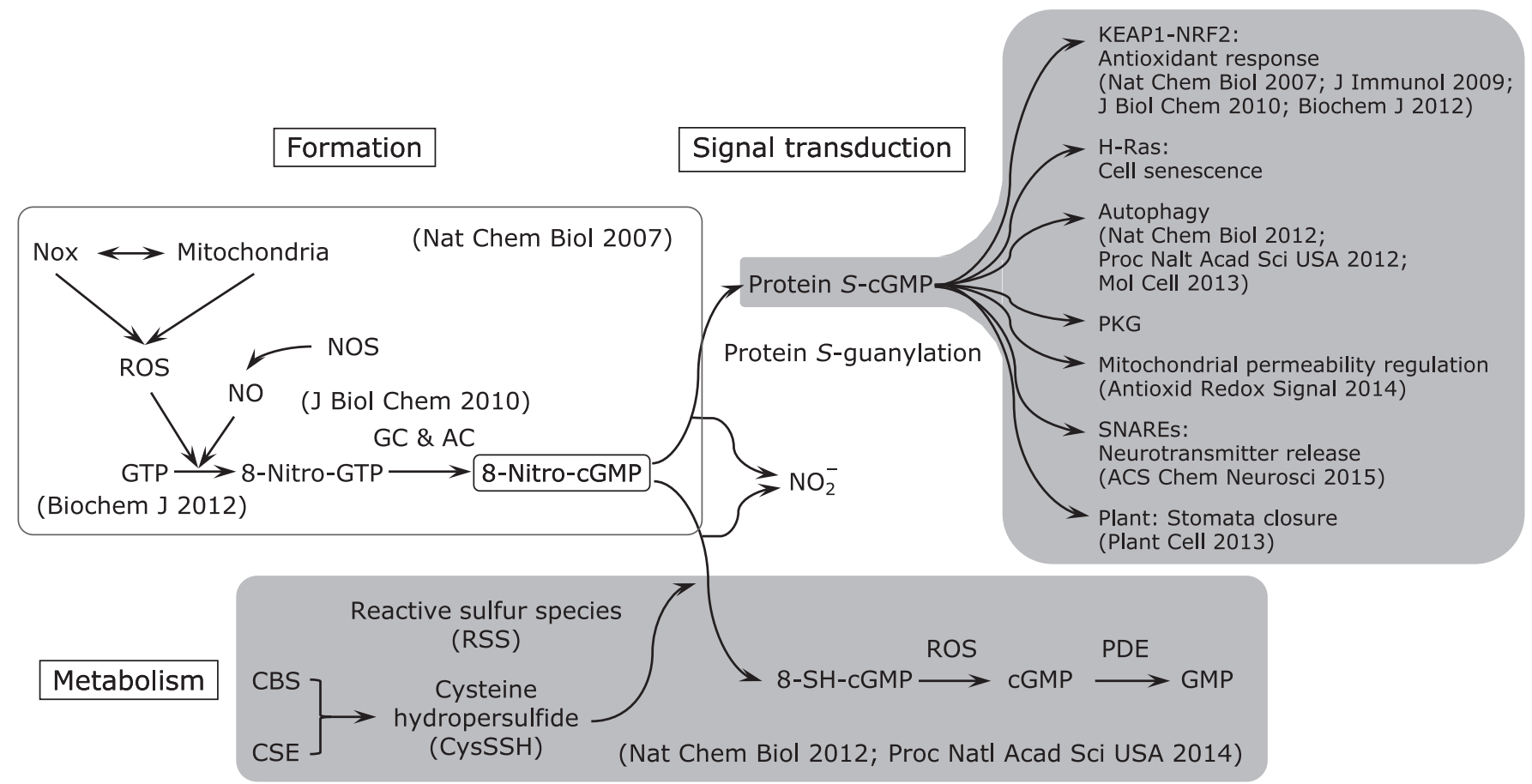

Fig. 2. Formation, signal transduction, and metabolism of 8-nitro-cGMP. PDE, phosphodiesterase; PKG, protein kinase G. 
in structures of the proteins, which often serve simultaneously as sensors and effectors (Fig. 1 and 2). Phosphorylation and transcriptional signaling are regulated via redox-sensitive structural changes in sensor-effector proteins including protein kinases, phosphatases, and transcription factors, with these changes being mostly caused by chemical modifications, e.g., oxidation, nitrosylation, alkylation, and guanylation, of cysteine sulfhydryls by ROS and reactive nitrogen oxide species as well as electrophilic second messengers such as 8-nitro-cGMP. ${ }^{(3)}$ Elucidating the sensor-effector relationship with ROS is of great importance in ROS research. In fact, novel mechanisms of ROS-dependent nuclear factor- $\kappa \mathrm{B}$ activation, ${ }^{(22)} S$-nitrosylation-mediated regulation of phosphatidylinositol-3-kinase-Akt signaling, ${ }^{(23)}$ and angiotensin type 1 receptor expression ${ }^{(24)}$ were recently clarified in the context of such a redox sensor-effector relationship. Exploring redox-based signal regulation will promote greater understanding of cellular response mechanisms, e.g., cell proliferation and cell death, mediated by ROS signaling and involving phosphorylation signaling, transcriptional regulation, endoplasmic reticulum stress, and neuronal and vascular signal transduction (Fig. 1).

\section{8-Nitro-cGMP as an Electrophilic Messenger for ROS and NO}

8-Nitro-cGMP biosynthesis. We found in 2007 that the nitrated guanine nucleotide 8-nitro-cGMP forms in cells (Fig. 2) and plays a crucial role in ROS signaling via a unique posttranslational modification (PTM) of cysteine residues in proteins, with the PTM process being called protein $S$-guanylation. ${ }^{(11)} \mathrm{We}$ verified that peroxynitrite $\left(\mathrm{ONOO}^{-}\right)$, which is produced by cellular expression of both $\mathrm{O}_{2}^{-}$and $\mathrm{NO}$, is the molecular species that is most likely responsible for nitration of guanine nucleotides and that nitrite plus $\mathrm{H}_{2} \mathrm{O}_{2}$ and myeloperoxidase may result in nitration of guanine nucleotides in a specific molecular environment in cells. ${ }^{(12,25)}$ We extensively analyzed the cellular formation of 8nitro-cGMP by using rat $\mathrm{C} 6$ glioma cells that produce $\mathrm{O}_{2}^{-{ }^{-}}$and $\mathrm{NO}$ in response to stimulation with lipopolysaccharide plus proinflammatory cytokines via Nox 2 and the inducible isoform of NO synthase (iNOS), respectively. ${ }^{(11,16)}$ In these stimulated cells, we identified mitochondria-derived $\mathrm{O}_{2}{ }^{--}$as a direct determinant of 8nitro-cGMP formation. Mitochondria-derived $\mathrm{O}_{2}{ }^{--}$production is itself regulated by $\mathrm{H}_{2} \mathrm{O}_{2}$ generated by Nox 2 and plays a critical role in 8-nitro-cGMP formation, which suggests that an essential link exists between Nox2-dependent $\mathrm{H}_{2} \mathrm{O}_{2}$ production and mitochondrial $\mathrm{O}_{2}{ }^{--}$production (Fig. 1). This finding may lend credence to our proposal that 8-nitro-cGMP may serve as a unique second messenger that forms downstream of $\mathrm{NO}$ and ROS generation. It is worth noting that 8-nitro-cGMP itself further augments mitochondria-derived $\mathrm{O}_{2}^{-{ }^{-}}$production (Fig. 1).(26) $S$-Guanylation of mitochondrial heat shock proteins including HSP60 seems to be involved in this enhanced ROS production, possibly by activating mitochondrial permeability transition pore opening, ${ }^{(26)}$ which suggests that 8-nitro-cGMP and mitochondria form a positive feed-forward loop to amplify ROS production.

The exact amount of 8-nitro-cGMP formed endogenously was determined by means of a quantitative analytical system for 8nitro-cGMP that utilized liquid chromatography tandem mass spectrometry (LC-MS/MS) with C6 cells stimulated as mentioned above. Our analysis determined that quite high concentrations $(>40 \mu \mathrm{M})$ of 8-nitro-cGMP occur in cells and that these levels are much higher than those of cGMP $(4.6 \mu \mathrm{M})$ in the same cells, which indicates that 8-nitro-cGMP is a major cyclic nucleotide generated in cells. ${ }^{(16)}$ Our quantitative LC-MS/MS analysis also revealed that 8-nitro-cGMP is formed by guanylate cyclasedependent cyclization of 8-nitroguanosine 5'-triphosphate (8nitro-GTP), which is generated during nitration of the abundant GTP, rather than being formed by direct nitration of cGMP (Fig. 2). Among different guanine nucleotides including cGMP,
GTP is the most susceptible to $\mathrm{ONOO}^{-}$-mediated nitration. ${ }^{(12)}$ More important, the 8-nitro-GTP formed by the reaction of GTP with $\mathrm{ONOO}^{-}$can be an efficient substrate for soluble guanylate cyclase to form 8-nitro-cGMP. ${ }^{(16)}$

8-Nitro-cGMP formation was also identified in vivo, e.g., in heart and brain tissues in mice. ${ }^{(13,20)}$ ROS and NO generated from Nox2 and iNOS, respectively, have been implicated in the pathogenesis of heart failure. ${ }^{(27,28)}$ By using LC-MS/MS analysis and immunohistochemistry with an anti-8-nitro-cGMP-specific antibody, we showed that 8-nitro-cGMP also forms in mouse hearts after myocardial infarction (MI) or pressure overload. ${ }^{(13)}$ iNOSdeficient hearts evidence no 8-nitro-cGMP formation after MI, which suggests that the iNOS-derived NO may be essential for 8-nitro-cGMP formation in mouse hearts. Lipopolysaccharide or ATP, which reportedly generates NO through iNOS induction in heart cells, ${ }^{(24)}$ induces 8-nitro-cGMP formation and protein $S$ guanylation in cultured rat neonatal cardiac myocytes and fibroblasts. These results indicate that heart cells actively produce, in an iNOS-dependent manner, 8-nitro-cGMP in response to stimulation with different agonists. In a later section, we discuss the pathological roles of chronic 8-nitro-cGMP formation in heart failure.

8-Nitro-cGMP metabolism by endogenous thiol-containing nucleophiles. The electrophilicity of 8 -nitro-cGMP is much lower than that of other endogenous electrophiles in general. Therefore, the relative stability of 8-nitro-cGMP in cells is a wellcharacterized chemical and pharmacological feature. For example, the second-order rate constant for the reaction of 8-nitro-cGMP with the glutathione (GSH) sulfhydryl at $\mathrm{pH} 7.4$ and $37^{\circ} \mathrm{C}$ is $0.03 \mathrm{M}^{-1} \mathrm{~s}^{-1}$. ${ }^{(11,29)}$ Also, 8-nitro-cGMP is much less reactive than other endogenous electrophiles including $\alpha, \beta$-unsaturated aldehydes, $\omega-6$ and $\omega-3$ unsaturated fatty acids, and nitroalkene fatty acids such as 4-hydroxy-2-nonenal, 15-deoxy- $\Delta^{12,14}$-prostaglandin $\mathrm{J}_{2}\left(15 \mathrm{~d}-\mathrm{PGJ}_{2}\right)$, and nitrolinoleic and nitrooleic acids. The secondorder rate constants for the reaction of those electrophiles with $\mathrm{GSH}$ at $\mathrm{pH} 7.4$ and $37^{\circ} \mathrm{C}$ are $1.3 \mathrm{M}^{-1} \mathrm{~s}^{-1}$ (4-hydroxy-2-nonenal), $0.7 \mathrm{M}^{-1} \mathrm{~s}^{-1}\left(15 \mathrm{~d}-\mathrm{PGJ}_{2}\right), 355 \mathrm{M}^{-1} \mathrm{~s}^{-1}$ (nitrolinoleic acid), and $183 \mathrm{M}^{-1} \mathrm{~s}^{-1}$ (nitrooleic acid). ${ }^{(29-31)}$ These rate constants thus indicate that these electrophiles react with the GSH sulfhydryl 20-10,000 times more rapidly than does 8-nitro-cGMP. ${ }^{(29)} 8$-NitrocGMP therefore has enough stability to remain at appreciable levels in cells even with excessive amounts of GSH, and it can affect its targets effectively even far from the site of 8-nitro-cGMP formation.

Detoxification of some electrophiles with GSH is facilitated by enzymes such as glutathione $S$-transferase (GST), which in fact plays an important role in eliminating reactive electrophiles generated during oxidative stress. ${ }^{(32)}$ No obvious degradation of 8-nitro-cGMP in the catalytic reaction with GST was observed, however. This remarkable biochemical characteristic of 8-nitrocGMP supports its high stability and its biological availability for redox signaling in cells.

Reactive cysteine persulfides as nucleophilic regulators of 8-nitro-cGMP metabolism and redox signaling. Our recent studies revealed endogenous formation of highly nucleophilic cysteine-based hydropersulfide species in biological systems. ${ }^{(33)}$ We found that cystathionine $\beta$-synthase (CBS) and cystathionine $\gamma$-lyase (CSE) are involved in 8-nitro-cGMP metabolism. ${ }^{(13)}$ Extensive biochemical and LC-MS/MS analyses showed that these two enzymes produce cysteine hydropersulfide from cystine used as a substrate. ${ }^{(33)}$ This cysteine hydropersulfide is highly nucleophilic and a stronger antioxidant than is cysteine or GSH (Fig. 3). This unique chemical property of reactive cysteine persulfide relies on adjacent electron pairs, an effect that is known as the $\alpha$-effect. ${ }^{(34,35)}$ Because of its chemical reactivity that depends on strong nucleophilicity, cysteine hydropersulfide can behave as a typical RSS and reacts quite effectively with 8-nitro-cGMP to form 8-SH-cGMP, with the release of a nitrite anion (Fig. 2), so that RSS such as cysteine hydropersulfide are implicated in 


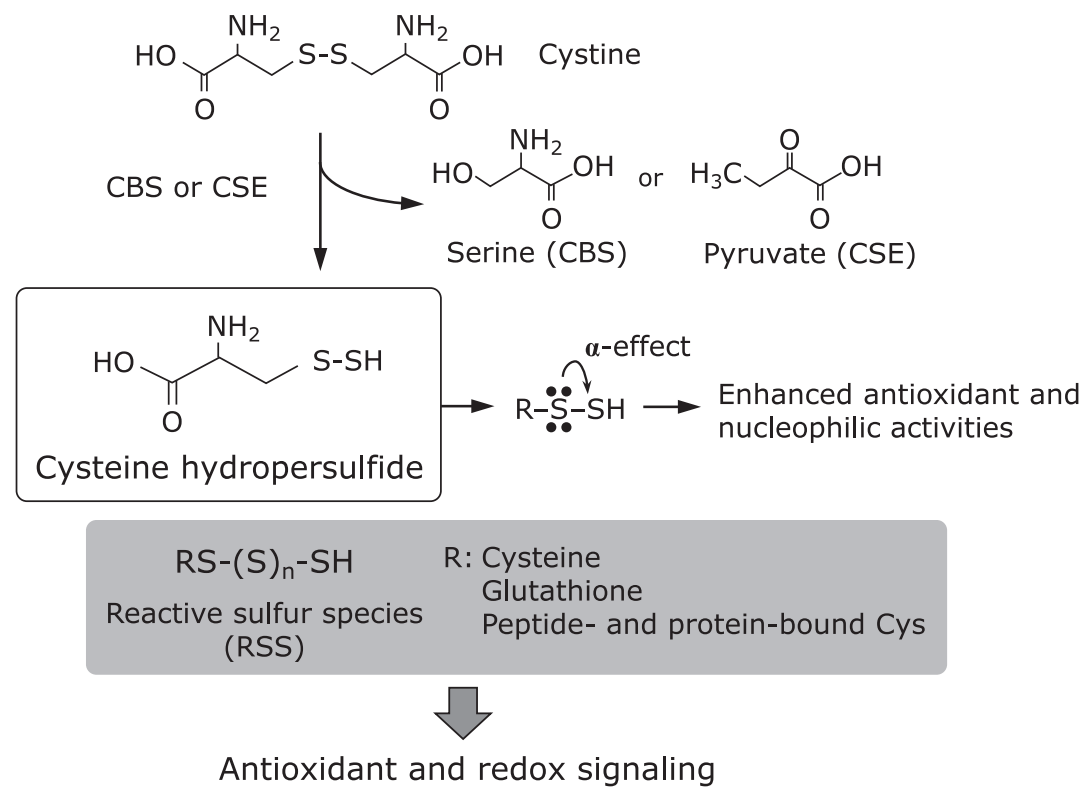

Fig. 3. RSS formation mediated by CBS and CSE. After RSS form, the antioxidant and nucleophilic activities of their terminal thiol residues are enhanced by an effect brought about by lone-pair electrons of adjacent sulfur atoms. CBS, cystathionine $\beta$-synthase; CSE, cystathionine $\gamma$-lyase.

the regulation ROS signaling. As Fig. 2 illustrates, two unique second messengers endogenously generated as affected by cysteine hydropersulfide may mediate signal transduction in a manner opposite to their redox properties, i.e., electrophilic 8-nitro-cGMP and nucleophilic 8-SH-cGMP. In fact, 8-SH-cGMP formation was identified as occurring in cultured mammalian cells, in mice, as well as in plant cells. . $^{(13,19,33)}$

The importance of RSS in the regulation of redox and electrophilic signaling is bolstered by our recent observation that these reactive persulfide species exist in all organs at appreciable concentrations. In fact, we successfully confirmed the endogenous formation of persulfides as cysteine, homocysteine, and GSH in mouse heart, liver, brain, kidney, spleen, small intestine, lung, and eye. ${ }^{(33)}$ Another intriguing finding is that RSS or reactive cysteine persulfide residues are present in various proteins, although the ubiquitous and possible regulatory function associated with protein persulfides and polysulfides remains unclear. ${ }^{(33)}$

\section{Electrophilic Cell Signaling Mediated by 8-Nitro-cGMP}

\section{Specificity of cell signaling conferred by 8-nitro-cGMP.}

8-Nitro-cGMP serves as an electrophilic second messenger because of its unique chemical reactivity. In fact, 8-nitro-cGMP undergoes nucleophilic substitution with a protein sulfhydryl, releases the nitro group, and forms a protein Cys-cGMP adduct in a process called protein $S$-guanylation. ${ }^{(11)} P$ Potein $S$-guanylation as a PTM occurring in cells is a major focus of our present studies, because identification of the physiological relevance of $S$ guanylation may promote research on oxidative stress and redox signaling. ${ }^{(36)}$

The low electrophilicity of 8-nitro-cGMP may be essential for the specificity of 8-nitro-cGMP in protein $S$-guanylationdependent redox signal transduction. Because of highly reactive electrophiles such as nitro-fatty acids, nucleophilic amino acids other than cysteine, especially histidine and lysine, also become targets in electrophilic reactions via Michael additions, ${ }^{(10)}$ during which they undergo unstable, reversible $S$-alkylation in a process called transalkylation. ${ }^{(37)}$ As signaling molecules with significant effects on cysteine thiols of acceptor proteins, therefore, reactive electrophiles may require a specific reaction environment or compartmentalization. Certain unique structural characteristics may be required for the stable covalent binding in electrophilic protein $S$-alkylation occurring in the vicinity of PTM sites. Because of the inert chemical reactivity of 8-nitro-cGMP as a physiological electrophile, however, $S$-guanylation occurs with sulfhydryls that have high nucleophilicity, as determined by the $\mathrm{p} K_{\mathrm{a}}$ of the cysteine sulfhydryl moiety. We identified protein targets for $S$-guanylation that are critically involved in the regulation of redox cellular signaling, as discussed in detail below.

Antioxidant responses induced by $S$-guanylation of Kelch-like ECH-associated protein 1 (KEAP1). The KEAP1nuclear factor-erythroid-2-related factor 2 (NRF2) system has pivotal roles in the mechanism of the stress response to oxidative and electrophilic insults by means of activating a battery of cytoprotective genes. These gene products are involved in GSH synthesis, NADPH production, scavenging ROS, xenobiotic detoxification, and cross-membrane transport that often results in multidrug resistance of cancers. ${ }^{(38-40)}$ NRF2 is a potent transcriptional activator responsible for induction of these antioxidant cytoprotective genes, whereas KEAP1 is an inhibitory regulator of NRF2. Under normal conditions, NRF2 is constantly ubiquitinated by KEAP1 and degraded by proteasomes. In the presence of electrophiles or ROS, KEAP1 is inactivated, and NRF2 is stabilized, binds to antioxidant response elements, and induces the cytoprotective genes. The critical contribution of NRF2 to antioxidant responses has been demonstrated in mice and humans. Nrf2-deficient mice are susceptible to oxidative stress, both exogenous and endogenous. A single nucleotide polymorphism in the promoter region of the mouse $N r f 2$ gene is associated with reduced expression of the $N r f 2$ gene and with susceptibility to hyperoxic lung injury in the C57BL/6J mouse. ${ }^{(41)}$ Single nucleotide polymorphisms in the promoter region of the human NRF2 gene $^{(42)}$ are linked to a higher risk of acute lung injury. ${ }^{(43)}$

The most characteristic feature of NRF2 function is its inducibility: NRF2 is active only when its negative regulator KEAP1 is inactivated in response to stimuli. KEAP1, possessing multiple reactive cysteine residues, serves as a biosensor for stimuli including electrophiles, ROS, reactive nitrogen oxides, and heavy metals, which directly modify cysteine residues of KEAP1. Distinct sets of cysteine residues are responsible for sensing distinct 
electrophiles. ${ }^{(44-46)}$ Substitution of the Cys151 of KEAP1 with serine abrogates the reactivity to a specific set of electrophiles including sulforaphane, dimethyl fumarate, and NO. However, this substitution does not affect the reactivity to nitro-fatty acids, ebselen, or $15 \mathrm{~d}_{-} \mathrm{PGJ}_{2}$, for which Cys273/288 residues have been suggested as alternative sensor cysteines. An interesting finding is that 8-nitro-cGMP, which operates as part of the NO signaling system, covalently modifies KEAP1 at Cys434 because of its electrophilic nature, which results in $S$-guanylation of KEAP1 and consequent activation of NRF2. ${ }^{(11,16)}$ Thus, specific cysteine residues are critical for the biosensor function of KEAP1. Clarification of the effect of RSS and polythiolation of KEAP1 cysteine residues may be an intriguing challenge and may help understanding of a fundamental mechanism: how organisms utilize sulfur to sense electrophiles.

Redox signaling by H-Ras activation via S-guanylation.

Most typically, cardiac remodeling appears to be modulated by electrophilic signaling mediated by 8-nitro-cGMP. For example, induction of early myocardial senescence is a major risk that exacerbates mortality and cardiac dysfunction after MI. Oxidative stress, nucleotide damage (e.g., by UV irradiation), and abnormal activation of oncogenes, including Ras family $G$ proteins, have reportedly mediated cellular senescence. ${ }^{(47,48)}$ Formation of ROSor RNS-derived electrophilic species such as $15 \mathrm{~d}_{-} \mathrm{PGJ}_{2}$ during an oxidative inflammatory response activates H-Ras through covalent modification of cysteine thiols, ${ }^{(49,50)}$ which in turn may activate p53-dependent cellular senescence. ${ }^{(47,48)} \mathrm{H}$-Ras contains two reactive cysteine residues. One is Cys 118 located in a guanine nucleotide-binding site, which is reportedly modified by ROS and reactive nitrogen oxide species. ${ }^{(50)}$ However, neuronal NOSmediated $S$-nitrosylation of $\mathrm{H}$-Ras at Cys 118 reportedly suppressed $\mathrm{H}$-Ras activity induced by a $\mathrm{Ca}^{2+}$ ionophore, ${ }^{(51)}$ which indicates that modification of the Cys118 on Ras does not necessarily activate Ras-dependent signaling pathways.

The other residue is Cys184, one of two palmitoylation sites located at the H-Ras carboxyl-terminal domain. ${ }^{(52)}$ We found that 8-nitro-cGMP, an oxidized nucleotide, increases H-Ras activity via specific $S$-guanylation of Cys 184 in mouse hearts with chronic heart failure. In contrast, other electrophiles, such as 1,2-naphthoquinone $(1,2-\mathrm{NQ})$ and methylmercury $(\mathrm{MeHg})$, do not modify the Cys 184 of H-Ras or induce H-Ras activation, which suggests that 8-nitro-cGMP may function as a specific physiological ligand for $\mathrm{H}-\mathrm{Ras}$ activation. Palmitoylation of Ras predominantly regulates subcellular localization of $\mathrm{H}$-Ras and efficiently activates a local effector (i.e., Raf-1)-mediated signaling pathway. ${ }^{(52)}$ Monopalmitoylation of Cys 181 contributes to efficient trafficking of H-Ras to the plasma membrane, and GDP-bound H-Ras is predominantly localized on lipid rafts. GTP loading of H-Ras dissociates H-Ras from the rafts, which results in the efficient association with Raf in the vicinity of the plasma membrane. Cys184 is not essential for targeting of H-Ras to the plasma membrane, but it is indispensable for control of GTP-regulated lateral segmentation of H-Ras between lipid rafts and non-rafts. Thus, $S$-guanylation of H-Ras at Cys184 may promote localization of H-Ras to the plasma membrane and association with Raf by dissociating H-Ras from lipid rafts. The $S$-guanylated H-Ras continuously activates downstream phosphorylation signals including ERK (extracellular signal-regulated kinase) and p38 mitogen-activated protein kinase, which leads to induction of $\mathrm{p} 53 / \mathrm{Rb}$ (retinoblastoma gene product)mediated myocardial cell senescence and thereby results in the transition of the heart condition from hypertrophy to heart failure.

Furthermore, long-term treatment with low doses $(50 \mu \mathrm{mol} / \mathrm{kg}$ / day) of sodium hydrosulfide (NaHS) suppresses $S$-guanylation and activation of H-Ras, myocardial cell senescence, and left ventricular dysfunction in mouse hearts after MI by eliminating 8-nitro-cGMP accumulation. ${ }^{(13)}$ However, NaHS hardly eliminates electrophiles in vitro, which indicates that NaHS may contribute to the formation in cells of more nucleophilic RSS, such as cysteine persulfide and dihydropersulfide, ${ }^{(33,53)}$ and may thus act as a thiol substrate so that endogenous electrophiles are eliminated in the failing heart. In view of the fact that a strong reducing reagent, 2-mercaptoethanol, partially reverses $S$-guanylation of H-Ras, formation of cysteine persulfide or $S$-polythiolation of H-Ras at Cys184 determines the reversibility of redox signaling mediated by 8-nitro-cGMP and may predominantly regulate the transition of the heart from adaptive hypertrophy to heart failure.

\section{Capture of RSS Formed in Metabolites and Proteins by Exogenous Electrophiles}

Various exogenous electrophiles occur in the environment. For example, 1,2-NQ, a contaminant in atmospheric particulate matter (PM2.5) and in cigarette smoke, is produced during photooxidation of naphthalene. $\mathrm{MeHg}$ accumulates in fish (e.g., tuna) through biocondensation. Although these environmental electrophiles cause deleterious effects in the body, we found that 1,2-NQ and $\mathrm{MeHg}$ activate redox-dependent cell signaling pathways such as protein tyrosine phosphatase $1 \mathrm{~B}$ /epidermal growth factor receptor signaling and KEAP1-NRF2 pathways via covalent modification of the reactive thiol groups of protein tyrosine phosphatase $1 \mathrm{~B}$ and KEAP1, respectively, at nontoxic concentrations. ${ }^{(54-56)}$ Activation of these redox signaling pathways associated with cellular protection causes elevation of the threshold for environmental electrophile-mediated cell damage. ${ }^{(57)}$

After MeHg invades cells, it undergoes conjugation with GSH produced by glutamate-cysteine ligase, after which the polar metabolite MeHg-SG is excreted into the extracellular space by means of multidrug resistance-associated protein. ${ }^{(58)}$ Experiments with $N r f 2$-deficient mice and primary mouse hepatocytes revealed that NRF2 is a transcription factor that has a role in the reduction of $\mathrm{MeHg}$ toxicity in vitro and in vivo, ${ }^{(56,59)}$ because NRF2 cooperatively regulates glutamate-cysteine ligase and multidrug resistance-associated protein. ${ }^{(60)}$ However, our recent study showed that the MeHg-SG adduct readily reacts with cellular proteins that have reactive thiol groups, including KEAP1, through $S$-transmercuration, which results in substantial cytotoxicity in SH-SY5Y cells. ${ }^{(61)}$ This finding suggests that although the MeHg-SG adduct, as well as $\mathrm{MeHg}$, can activate NRF2 coupled to the modification of KEAP1, an increase in $\mathrm{MeHg}$ bound proteins is associated with cellular toxicity. In other words, a detoxification pathway or pathways other than NRF2 for $\mathrm{MeHg}$ seems likely.

Several researchers who are interested in endogenous gas signaling molecules, such as NO and carbon monoxide, have believed that endogenous hydrogen sulfide $\left(\mathrm{H}_{2} \mathrm{~S}\right)$ also plays a role in regulating neurotransmission and vascular tone. ${ }^{(62)}$ However, the $\mathrm{p} K_{\mathrm{a}}$ value of $\mathrm{H}_{2} \mathrm{~S}$ is 6.76 , which indicates that under physiological conditions approximately $80 \%$ of $\mathrm{H}_{2} \mathrm{~S}$ exists as the deprotonated $\mathrm{HS}^{-}$anion. We therefore postulated that environmental electrophiles such as $\mathrm{MeHg}$ readily undergo nucleophilic attack by the $\mathrm{HS}^{-}$anion, which leads to formation of sulfur adducts [e.g., the MeHg-SH adduct and/or bismethylmercury sulfide $\left.(\mathrm{MeHg})_{2} \mathrm{~S}\right]$. In fact, we isolated a previously unknown metabolite of $\mathrm{MeHg}$ from $\mathrm{SH}-\mathrm{SY} 5 \mathrm{Y}$ cells exposed to $\mathrm{MeHg}$ and from the liver of rats given $\mathrm{MeHg}$, which we identified as $(\mathrm{MeHg})_{2} \mathrm{~S} .{ }^{(63)}$ Furthermore, an in vivo study with authentic $(\mathrm{MeHg})_{2} \mathrm{~S}$ showed that this sulfur adduct is a detoxified metabolite of MeHg. ${ }^{(63)} \mathrm{A}$ separate study demonstrated that the $1,2-\mathrm{NQH}_{2}-\mathrm{SH}$ adduct (detected as the oxidized 1,2-NQ-SH adduct) forms during interaction of 1,2-NQ with $\mathrm{H}_{2} \mathrm{~S} / \mathrm{HS}^{-}$through a 1,4-Michael addition reaction, ${ }^{(13)}$ which suggests that this sulfur adduct may act as a signaling molecule to regulate redox-dependent cell signaling pathways. Consistent with this idea, the $1,2-\mathrm{NQH}_{2}-\mathrm{SH}$ adduct was also shown to activate the KEAP1-NRF2 pathway through sulfenic acid formation, followed by KEAP1-S-S-1,2-NQH $\mathrm{N}_{2}$ production. ${ }^{(64)}$ This finding is an example of RSS-mediated reversible 


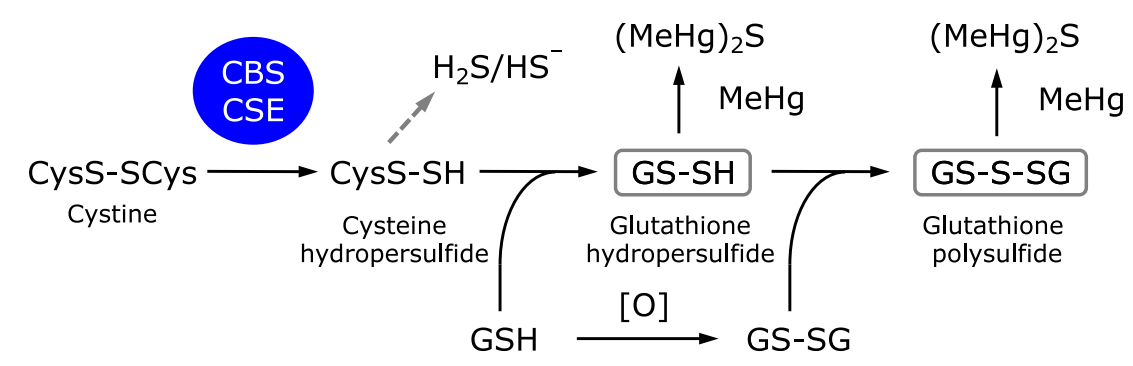

Protein-bound RSS

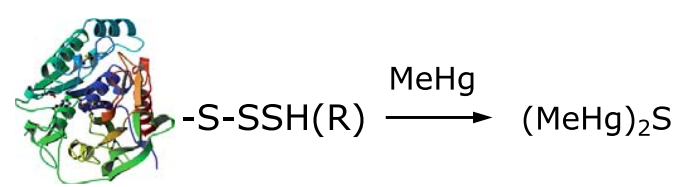

Fig. 4. Formation of bismethylmercury sulfide during interaction with RSS. CBS, cystathionine $\beta$-synthase; CSE, cystathionine $\gamma$-lyase; GSH, glutathione; GS-SG, oxidized glutathione; $\mathrm{H}_{2} \mathrm{~S}$, hydrogen sulfide; $\mathrm{HS}^{-}$, hydrogen sulfide anion; (MeHg) ${ }_{2} \mathrm{~S}$, bismethylmercury sulfide; MeHg, methylmercury.

electrophilic signaling under oxidative stress.

In addition, our recent experiments clearly demonstrated that CBS and CSE mainly catalyze the formation of cysteine hydropersulfide from cystine, but with little appreciable production of $\mathrm{H}_{2} \mathrm{~S} / \mathrm{HS}^{-}{ }^{(33)}$ Of interest, this cysteine hydropersulfide reacts spontaneously with GSH, the result being the production of GSH hydropersulfide, which is oxidized to GSH polysulfide. (33) This finding suggests that $(\mathrm{MeHg})_{2} \mathrm{~S}$ may form in vitro and in vivo during the interaction of $\mathrm{MeHg}$ with these RSS. Cell-free experiments with RSS and synthetic polysulfides, such as $\mathrm{Na}_{2} \mathrm{~S}_{4}$, indicated that $(\mathrm{MeHg})_{2} \mathrm{~S}$ is the common product after reaction with $\mathrm{NaHS}$, GSH hydropersulfide, GSH polysulfide, $\mathrm{Na}_{2} \mathrm{~S}_{4}$, and even protein-bound reactive persulfide/polysulfide species, ${ }^{(65)}$ as Fig. 4 shows. In addition to $\mathrm{MeHg}$, we also found that sulfur adducts of $N$-acetyl- $p$-benzoquinone imine, an electrophilic metabolite of acetaminophen, are produced in the biological samples of mice upon acetaminophen exposure. ${ }^{(66)}$ For these reasons, we thought that an $(\mathrm{MeHg})_{2} \mathrm{~S}$ formation assay would be a convenient method to identify proteins with reactive persulfides or polysulfides. Although Cibacron Blue 3GA column chromatography of mouse liver cytosol revealed many proteins with reactive persulfide or polysulfide moieties, as mentioned in our previous study that utilized the Tag-Switch-Tag assay, ${ }^{(33)}$ subsequent Sephacryl S-100 column chromatography showed a highly purified $25-\mathrm{kDa}$ protein to be GST P1. ${ }^{(65)}$ Incubation of recombinant GST P1 with $\mathrm{MeHg}$ confirmed that this protein certainly contains reactive persulfides or polysulfides bound to the cysteine residues.

Little doubt remains that $(\mathrm{MeHg})_{2} \mathrm{~S}$ is produced as a result of the capture by $\mathrm{MeHg}$ of a mobilized sulfur atom from endogenous RSS and protein-bound RSS (Fig. 4). Because RSS have high nucleophilicity and antioxidant capability, substantial $(\mathrm{MeHg})_{2} \mathrm{~S}$ formation is associated with a reduction in nucleophilic and/or reductive cellular status. Thus, we propose a new concept: RSS protect cells from electrophilic insults, and excessive exposure to electrophilic species appears to deplete these RSS, which leads to greater susceptibility to electrophile-dependent toxicity.

\section{Conclusion}

Redox signaling is precisely regulated by various sensor and effector molecules. 8-Nitro-cGMP is an endogenous electrophile with physiological functions and possesses a unique property contributing to its specificity and selectivity for ROS signal transduction. 8-Nitro-cGMP may thus be able to serve as a natural endogenous ROS ligand that can induce specific cellular responses through $S$-guanylation of specific cysteine residues in redox sensor-effector proteins, such as KEAP1 and H-Ras. Recent progress in identification of RSS formed endogenously revealed that RSS such as reactive cysteine persulfides found in abundant amounts in cells possess markedly highly antioxidant and nucleophilic properties. These RSS are critically involved not only in detoxification of environmental electrophiles but also in regulation of ROS signaling by means of 8-nitro-cGMP metabolism. Evidence of protein $S$-guanylation induced by 8-nitro-cGMP and regulation of ROS signaling by RSS may thus warrant further innovative research that may reveal new aspects of ROS- and RSS-related redox chemical biology, physiology, pathophysiology, and pharmaceutical chemistry and may promote the development of novel therapeutics for oxidative stress-related diseases.

\section{Acknowledgments}

We thank J.B. Gandy for her excellent editing of the manuscript. We gratefully thank Profs. M. Yamamoto, K. Uchida, and T. Sawa, and other Principal Investigators who were responsible for the 12 research areas of the program project entitled "Signaling Functions of Reactive Oxygen Species," which was supported by Grants-in-Aid for Scientific Research on Innovative Areas (Research in a Proposed Research Area) from the Ministry of Education, Culture, Sports, Science and Technology (MEXT), Japan, from 2008 to 2012, for their invaluable discussions and collaboration. This work was supported in part by Grants-in-Aid for Scientific Research (B) to M.N. (No. 25293018), (S) to Y.K. (No. 25220103), (C) to H.I. (No. 25430069), (C) to S.F. (No. 15K08456), (B) to H.M. (No. 15H04692), and (A) to T.A. (No. 25253020); by Grants-in-Aid for Scientific Research on Innovative Areas (Research in a Proposed Research Area) to T.A., H.M., M.N., H.I., and Y.K., from the Ministry of Education, Culture, Sports, Science and Technology (MEXT); and by PRESTO, Japan Science and Technology Agency. 


\section{Abbreviations}

CBS
CSE
$15 \mathrm{~d}-\mathrm{PGJ}_{2}$
GSH
GST
iNOS
KEAP1
LC-MS/MS
MeHg
$(\mathrm{MeHg})_{2} \mathrm{~S}$
$\mathrm{MI}$

cystathionine $\beta$-synthase

cystathionine $\gamma$-lyase

15-deoxy- $\Delta^{12,14}$-prostaglandin $\mathrm{J}_{2}$ glutathione

glutathione $S$-transferase

inducible NO synthase

Kelch-like ECH-associated protein 1

liquid chromatography tandem mass spectrometry methylmercury

bismethylmercury sulfide

myocardial infarction

\section{References}

1 Beckman JS, Beckman TW, Chen J, Marshall PA, Freeman BA. Apparent hydroxyl radical production by peroxynitrite: implications for endothelial injury from nitric oxide and superoxide. Proc Natl Acad Sci U S A 1990; 87: $1620-1624$.

2 van der Vliet A, Cross CE. Oxidants, nitrosants, and the lung. Am J Med 2000; 109: 398-421.

3 Forman HJ, Fukuto JM, Torres M. Redox signaling: thiol chemistry defines which reactive oxygen and nitrogen species can act as second messengers. $\mathrm{Am}$ J Physiol Cell Physiol 2004; 287: C246-C256.

4 Halliwell B. Biochemistry of oxidative stress. Biochem Soc Trans 2007; 35 (Pt 5): 1147-1150.

5 Trachootham D, Alexandre J, Huang P. Targeting cancer cells by ROSmediated mechanisms: a radical therapeutic approach? Nat Rev Drug Discov 2009; 8: 579-591.

6 Sumimoto H. Structure, regulation and evolution of Nox-family NADPH oxidases that produce reactive oxygen species. FEBS $J$ 2008; 275: 3249 3277.

7 van der Vliet A. NADPH oxidases in lung biology and pathology: host defense enzymes, and more. Free Radic Biol Med 2008; 44: 938-955.

8 Matoba T, Shimokawa H, Nakashima M, et al. Hydrogen peroxide is an endothelium-derived hyperpolarizing factor in mice. J Clin Invest 2000; 106: 1521-1530.

9 Burgoyne JR, Madhani M, Cuello F, et al. Cysteine redox sensor in PKGI $\alpha$ enables oxidant-induced activation. Science 2007; 317: 1393-1397.

10 Schopfer FJ, Baker PR, Freeman BA. NO-dependent protein nitration: a cell signaling event or an oxidative inflammatory response? Trends Biochem Sci 2003; 28: 646-654.

11 Sawa T, Zaki MH, Okamoto T, et al. Protein $S$-guanylation by the biological signal 8-nitroguanosine 3',5'-cyclic monophosphate. Nat Chem Biol 2007; 3: $727-735$.

12 Ahmed KA, Sawa T, Ihara H, et al. Regulation by mitochondrial superoxide and NADPH oxidase of cellular formation of nitrated cyclic GMP: potential implications for ROS signalling. Biochem J 2012; 441: 719-730.

13 Nishida M, Sawa T, Kitajima N, et al. Hydrogen sulfide anion regulates redox signaling via electrophile sulfhydration. Nat Chem Biol 2012; 8: 714 724.

14 Schopfer FJ, Cipollina C, Freeman BA. Formation and signaling actions of electrophilic lipids. Chem Rev 2011; 111: 5997-6021.

15 Uchida K, Shibata T. 15-Deoxy- $\Delta^{12,14}$-prostaglandin $\mathrm{J}_{2}$ : an electrophilic trigger of cellular responses. Chem Res Toxicol 2008; 21: 138-144.

16 Fujii S, Sawa $\mathrm{T}$, Ihara $\mathrm{H}$, et al. The critical role of nitric oxide signaling, via protein $S$-guanylation and nitrated cyclic GMP, in the antioxidant adaptive response. J Biol Chem 2010; 285: 23970-23984.

17 Ito C, Saito Y, Nozawa T, et al. Endogenous nitrated nucleotide is a key mediator of autophagy and innate defense against bacteria. Mol Cell 2013; 52: 794-804.

18 Joudoi T, Shichiri Y, Kamizono N, et al. Nitrated cyclic GMP modulates guard cell signaling in Arabidopsis. Plant Cell 2013; 25: 558-571.

19 Honda K, Yamada N, Yoshida R, et al. 8-Mercapto-cyclic GMP mediates hydrogen sulfide-induced stomatal closure in Arabidopsis. Plant Cell Physiol 2015; 56: 1481-1489.

20 Kunieda K, Tsutsuki H, Ida T, et al. 8-Nitro-cGMP enhances SNARE complex formation through $S$-guanylation of Cys90 in SNAP25. ACS Chem Neurosci 2015; 6: 1715-1725.
8-nitro-cGMP 8-nitroguanosine 3',5'-cyclic monophosphate

8-nitro-GTP 8-nitroguanosine 5'-triphosphate

Nox NADPH oxidase

1,2-NQ 1,2-naphthoquinone

NRF2 nuclear factor-erythroid-2-related factor 2

PTM posttranslational modification

ROS reactive oxygen species

RSS reactive sulfur species

\section{Conflict of Interest}

No potential conflicts of interest were disclosed.

21 Akashi S, Ahmed AK, Sawa T, et al. Persistent activation of cGMPdependent protein kinase by a nitrated cyclic nucleotide via site-specific protein $S$-guanylation. Biochemistrty 2015; provisionally accepted.

22 Tsuchiya Y, Asano T, Nakayama K, Kato T Jr, Karin M, Kamata H. Nuclear $\mathrm{IKK} \beta$ is an adaptor protein for $\mathrm{I} \kappa \mathrm{B} \alpha$ ubiquitination and degradation in UVinduced NF- $\kappa$ B activation. Mol Cell 2010; 39: 570-582.

23 Numajiri N, Takasawa K, Nishiya T, et al. On-off system for PI3-kinase-Akt signaling through $S$-nitrosylation of phosphatase with sequence homology to tensin (PTEN). Proc Natl Acad Sci U S A 2011; 108: 10349-10354.

24 Nishida M, Ogushi M, Suda R, et al. Heterologous down-regulation of angiotensin type 1 receptors by purinergic $\mathrm{P}_{2} \mathrm{Y}_{2}$ receptor stimulation through $S$ nitrosylation of NF-кB. Proc Natl Acad Sci U S A 2011; 108: 6662-6667.

25 Radi R. Nitric oxide, oxidants, and protein tyrosine nitration. Proc Natl Acad Sci U S A 2004; 101: 4003-4008.

26 Rahaman MM, Sawa T, Ahtesham AK, et al. S-Guanylation proteomics for redox-based mitochondrial signaling. Antioxid Redox Signal 2014; 20: 295307.

27 Liu YH, Carretero OA, Cingolani $\mathrm{OH}$, et al. Role of inducible nitric oxide synthase in cardiac function and remodeling in mice with heart failure due to myocardial infarction. Am J Physiol Heart Circ Physiol 2005; 289: H2616H2623.

28 Krijnen PA, Meischl C, Hack CE, et al. Increased Nox2 expression in human cardiomyocytes after acute myocardial infarction. J Clin Pathol 2003; 56: 194-199.

29 Sawa T, Arimoto H, Akaike T. Regulation of redox signaling involving chemical conjugation of protein thiols by nitric oxide and electrophiles. Bioconjug Chem 2010; 21: 1121-1129.

30 Baker LM, Baker PR, Golin-Bisello F, et al. Nitro-fatty acid reaction with glutathione and cysteine. Kinetic analysis of thiol alkylation by a Michael addition reaction. J Biol Chem 2007; 282: 31085-31093.

31 Doorn JA, Petersen DR. Covalent adduction of nucleophilic amino acids by 4-hydroxynonenal and 4-oxononenal. Chem Biol Interact 2003; 143-144: 93100.

32 Hayes JD, Flanagan FU, Jowsey IR. Glutathione transferases. Annu Rev Pharmacol Toxicol 2005; 45: 51-88.

33 Ida T, Sawa T, Ihara $\mathrm{H}$, et al. Reactive cysteine persulfides and $S$-polythiolation regulate oxidative stress and redox signaling. Proc Natl Acad Sci U S A 2014; 111: 7606-7611.

34 Ono K, Akaike T, Sawa T, et al. Redox chemistry and chemical biology of $\mathrm{H}_{2} \mathrm{~S}$, hydropersulfides, and derived species: implications of their possible biological activity and utility. Free Radic Biol Med 2014; 77: 82-94.

35 Edwards JO, Pearson RG. The factors determining nucleophilic reactivities. J Am Chem Soc 1962; 84: 16-24.

36 Fujii S, Akaike T. Redox signaling by 8-nitro-cyclic guanosine monophosphate: nitric oxide- and reactive oxygen species-derived electrophilic messenger. Antioxid Redox Signal 2013; 19: 1236-1246.

37 Rudolph TK, Freeman BA. Transduction of redox signaling by electrophileprotein reactions. Sci Signal 2009; 2: re7.

38 Motohashi H, Yamamoto M. Nrf2-Keap1 defines a physiologically important stress response mechanism. Trends Mol Med 2004; 10: 549-557.

39 Uruno A, Motohashi H. The Keap1-Nrf2 system as an in vivo sensor for electrophiles. Nitric Oxide 2011; 25: 153-160.

40 Mitsuishi Y, Taguchi K, Kawatani Y, et al. Nrf2 redirects glucose and glutamine into anabolic pathways in metabolic reprogramming. Cancer Cell 2012; 
22: 66-79.

41 Cho HY, Jedlicka AE, Reddy SP, Zhang LY, Kensler TW, Kleeberger SR. Linkage analysis of susceptibility to hyperoxia. Nrf2 is a candidate gene. Am J Respir Cell Mol Biol 2002; 26: 42-51.

42 Yamamoto T, Yoh K, Kobayashi A, et al. Identification of polymorphisms in the promoter region of the human NRF2 gene. Biochem Biophys Res Commun 2004; 321: 72-79.

43 Marzec JM, Christie JD, Reddy SP, et al. Functional polymorphisms in the transcription factor NRF2 in humans increase the risk of acute lung injury. FASEB J 2007; 21: 2237-2246.

44 Kobayashi M, Li L, Iwamoto N, et al. The antioxidant defense system Keap1-Nrf2 comprises a multiple sensing mechanism for responding to a wide range of chemical compounds. Mol Cell Biol 2009; 29: 493-502.

45 McMahon M, Lamont DJ, Beattie KA, Hayes JD. Keap1 perceives stress via three sensors for the endogenous signaling molecules nitric oxide, zinc, and alkenals. Proc Natl Acad Sci U S A 2010; 107: 18838-18843.

46 Takaya K, Suzuki T, Motohashi H, et al. Validation of the multiple sensor mechanism of the Keap1-Nrf2 system. Free Radic Biol Med 2012; 53: 817 827.

47 Serrano M, Lin AW, McCurrach ME, Beach D, Lowe SW. Oncogenic ras provokes premature cell senescence associated with accumulation of p53 and p16 ${ }^{\mathrm{INK} 4 \mathrm{a}}$. Cell 1997; 88: 593-602.

48 Wu C, Miloslavskaya I, Demontis S, Maestro R, Galaktionov K. Regulation of cellular response to oncogenic and oxidative stress by Seladin-1. Nature 2004; 432: 640-645.

49 Lander HM, Milbank AJ, Tauras JM, et al. Redox regulation of cell signalling. Nature 1996; 381: 380-381.

50 Oliva JL, Pérez-Sala D, Castrillo A, et al. The cyclopentenone 15-deoxy$\Delta^{12,14}$-prostaglandin $\mathrm{J}_{2}$ binds to and activates H-Ras. Proc Natl Acad Sci U S A 2003; 100: 4772-4777.

51 Raines KW, Cao GL, Lee EK, Rosen GM, Shapiro P. Neuronal nitric oxide synthase-induced $S$-nitrosylation of H-Ras inhibits calcium ionophoremediated extracellular-signal-regulated kinase activity. Biochem J 2006; 397: 329-336.

52 Hancock JF. Ras proteins: different signals from different locations. Nat Rev Mol Cell Biol 2003; 4: 373-384.

53 Chen W, Rosser EW, Matsunaga T, Pacheco A, Akaike T, Xian M. The development of fluorescent probes for visualizing intracellular hydrogen polysulfides. Angew Chem Int Ed Engl 2015; in press. DOI: 10.1002/ anie. 201506887.
54 Iwamoto N, Sumi D, Ishii T, et al. Chemical knockdown of protein-tyrosine phosphatase $1 \mathrm{~B}$ by 1,2-naphthoquinone through covalent modification causes persistent transactivation of epidermal growth factor receptor. $\mathrm{J}$ Biol Chem 2007; 282: 33396-33404.

55 Miura T, Shinkai Y, Jiang HY, et al. Initial response and cellular protection through the Keap1/Nrf2 system during the exposure of primary mouse hepatocytes to 1,2-naphthoquinone. Chem Res Toxicol 2011; 24: 559-567.

56 Toyama T, Sumi D, Shinkai Y, et al. Cytoprotective role of Nrf2/Keap1 system in methylmercury toxicity. Biochem Biophys Res Commun 2007; 363: 645-650.

57 Kumagai Y, Shinkai Y, Miura T, Cho AK. The chemical biology of naphthoquinones and its environmental implications. Annu Rev Pharmacol Toxicol 2012; 52: 221-247.

58 Kumagai Y, Kanda H, Shinkai Y, Toyama T. The role of the Keap1/Nrf2 pathway in the cellular response to methylmercury. Oxid Med Cell Longev 2013; 2013: 848279.

59 Toyama T, Shinkai Y, Yasutake A, Uchida K, Yamamoto M, Kumagai Y. Isothiocyanates reduce mercury accumulation via an Nrf2-dependent mechanism during exposure of mice to methylmercury. Environ Health Perspect 2011; 119: 1117-1122.

60 Taguchi K, Motohashi H, Yamamoto M. Molecular mechanisms of the Keap1-Nrf2 pathway in stress response and cancer evolution. Genes Cells 2011; 16: 123-140.

61 Yoshida E, Abiko Y, Kumagai Y. Glutathione adduct of methylmercury activates the Keap1-Nrf2 pathway in SH-SY5Y cells. Chem Res Toxicol 2014; $27:$ 1780-1786

62 Gadalla MM, Snyder SH. Hydrogen sulfide as a gasotransmitter. J Neurochem 2010; 113: 14-26.

63 Yoshida E, Toyama T, Shinkai Y, Sawa T, Akaike T, Kumagai Y. Detoxification of methylmercury by hydrogen sulfide-producing enzyme in mammalian cells. Chem Res Toxicol 2011; 24: 1633-1635.

64 Shinkai Y, Abiko Y, Ida T, et al. Reactive sulfur species-mediated activation of the Keap1-Nrf2 pathway by 1,2-naphthoquinone through sulfenic acids formation under oxidative stress. Chem Res Toxicol 2015; 28: 838-847.

65 Abiko Y, Yoshida E, Ishii I, Fukuto JM, Akaike T, Kumagai Y. Involvement of reactive persulfides in biological bismethylmercury sulfide formation. Chem Res Toxicol 2015; 28: 1301-1306.

66 Abiko Y, Ishii I, Kamata S, et al. Formation of sulfur adducts of N-acetyl-pbenzoquinoneimine, an electrophilic metabolite of acetaminophen in vivo: Participarion of reactive persulfides. Chem Res Toxicol 2015; 28: 1796-1802. 